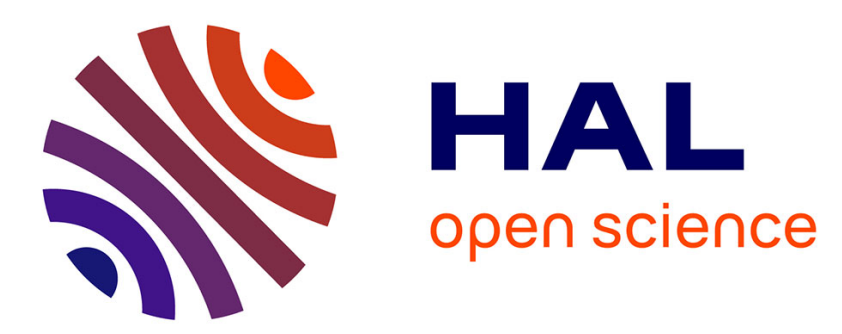

\title{
Interactive Competition Between Individual Diffusion Layers during Cyclic Voltammetry at Random Arrays of Band and Disk Electrodes. A Thorough Analysis Based on Global Simulations
}

Giovanni Pireddu, Irina Svir, Christian Amatore, Alexander Oleinick

\section{To cite this version:}

Giovanni Pireddu, Irina Svir, Christian Amatore, Alexander Oleinick. Interactive Competition Between Individual Diffusion Layers during Cyclic Voltammetry at Random Arrays of Band and Disk Electrodes. A Thorough Analysis Based on Global Simulations. ChemElectroChem, 2021, 10.1002/celc.202100310 . hal-03230372

\section{HAL Id: hal-03230372 \\ https://hal.science/hal-03230372}

Submitted on 19 May 2021

HAL is a multi-disciplinary open access archive for the deposit and dissemination of scientific research documents, whether they are published or not. The documents may come from teaching and research institutions in France or abroad, or from public or private research centers.
L'archive ouverte pluridisciplinaire HAL, est destinée au dépôt et à la diffusion de documents scientifiques de niveau recherche, publiés ou non, émanant des établissements d'enseignement et de recherche français ou étrangers, des laboratoires publics ou privés. 


\title{
Interactive Competition Between Individual Diffusion Layers during Cyclic Voltammetry at Random Arrays of Band and Disk Electrodes. A Thorough Analysis Based on Global Simulations."
}

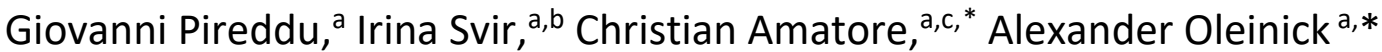

a Dr. G. Pireddu, Prof. Dr. I. Svir, Prof. Dr. C. Amatore, Dr. A. Oleinick, PASTEUR, Département de Chimie, École Normale Supérieure, PSL University, Sorbonne Université, CNRS, 24 rue Lhomond, 75005 Paris, France,

${ }^{b}$ Prof. Dr. I. Svir, Design Automation Department, Kharkiv National University of Radio Electronics, Nauky Avenue, 14, Kharkiv 61166, Ukraine,

c Prof. Dr. C. Amatore, State Key Laboratory of Physical Chemistry of Solid Surfaces, College of Chemistry and Chemical Engineering, Xiamen University, Xiamen 361005, China.

\begin{abstract}
This work validates a posteriori the use of the theory developed ca. 40 years ago by Amatore and Savéant for regular arrays of electroactive band or disk electrodes. This theory has been profitably used and widely applied in more than 900 research papers for the treatment of the voltammetric responses of arrays of nanoelectroactive objects that were necessarily random by construction. In this work we show that competitive diffusion interaction between the active sites enforce redistribution of individual diffusion layer which homogenize dimensions of the solution volumes 'feeding' each active site. For arrays of nanodisk electrodes, it also validates the corresponding approximation assuming that its voltammetric behavior is to that afforded by identical independent cylindrical unit cells performing in parallel.
\end{abstract}

Keywords: random arrays of electrodes; Voronoi tessellation; cyclic voltammetry; modelling; flux lines; simulations

\section{Introduction and preliminary historical considerations}

About 40 years ago, one of us (CA) published his last joint work with his mentor, Jean-Michel Savéant, on a subject that was then no more than a scientific curiosity ${ }^{[1]}$ Unexpectedly, this article on which no one would have dared to bet even a nickel, now collects more than 900 citations, currently ranking 2nd among Amatore citations and 3rd among Savéant. It therefore seemed particularly opportune to revisit this same topic with modern eyes as a contribution from our group in this special issue dedicated to honoring the memory of Jean-Michel Savéant.

At the time, the aim of this work was to offer a simple theoretical explanation for a fact experienced by any freshman or freshwoman in electrochemistry when dealing with voltammetry at solid electrodes: even when the polished metal surface appears already perfectly bright to the eye, voltammetric waves can appear sluggish vs. what is expected although they display flawless Cottrellian tails. Although this is never rationalized in textbooks, but learned the hard way in the lab, extra effort to polish the electrode gradually leads to "nicer" cyclic voltammograms, but without noticeable change in their Cottrellian tails. This demonstrated that the initial apparent waves slowness was not due to the presence of macroscopic adsorbed insulating patches. Indeed, the polishing would have

\footnotetext{
\# Dedicated to the memory of Prof. Dr. Jean-Michel Savéant who was mentor of one of us (CA).

*Corresponding authors: christian.amatore@ens.psl.eu ; oleksandr.oliynyk@ens.psl.eu
} 
then contributed to improving the current amplitudes of the whole voltammogram rather than reducing only the peak-to-peak potential gap. ${ }^{[2-5]}$

It quickly became clear that the only possible rationalization came down to attributing the surprising activity of partially polished electrodes to a network of microscopic electroactive sites distributed over its otherwise insulating surface and whose dimensions were considerably smaller than those of the voltammetric diffusion layer, viz., of the order of $(\pi D R T / F v)^{1 / 2}$ where $D$ is the electroactive species diffusion coefficient, $v$ the scan rate and $R, F$ and $T$ having their usual meaning (Perfect gas and Faraday constants and absolute temperature). ${ }^{[1,6,7]}$ However, in an immediately following experimental contribution, ${ }^{[8]}$ the same authors came to a seemingly unlikely conclusion at the time: these microscopic electroactive sites had to have nanometric dimensions in order to conform to the theory. At the time, electrochemistry had not yet fully entered in its microelectrode era, not to mention its current nanoscience era. Hence, such a result seemed totally improbable and was tamperproof beyond any question, being therefore almost a matter of faith.

Unexpectedly, 4 years later, three papers were published that confirmed the logic and experimental significance of these theoretical hypotheses. A first set of two daring papers by Rubinstein et al. ${ }^{[9,10]}$ concerned the voltammetric oxidation of $\mathrm{Fe}(\mathrm{CN})_{6}{ }^{4-}$ in $\mathrm{KCl}$ solutions at gold ultramicroelectrodes coated with carefully prepared supposedly compact SAMs consisting of mixtures of $n$-octadecyl trichlorosilane and $\mathrm{n}$-octadecyl mercaptan. These authors precisely observed the surprising outcome predicted in ref.[1] for arrays of nanometric active electrodes, viz., voltammetric waves with only very slightly reduced current amplitudes (ca. 10\% at most) but much higher peak-to-peak separations when referred to those recorded on the same bare gold ultramicroelectrodes before coating. Based on the Amatore and Savéant's theoretical predictions, Rubinstein et al. ascribed these unexpected observations to the presence of nanometric pinholes due to imperfections in the SAM coatings and could estimate their mean diameter to fall in the astonishing range of 5-10 nm with a mean spacing of 50-100 nm. Evidently, in 1987, the presence of nanometric pinholes in a SAM surface could not be directly observed in any electrochemical laboratories. Nonetheless, they were supported by similar results inferred from resistance measurements by impedance analyses of the same SAM-coated electrodes. This corroborated for the first time Amatore and Savéant's theoretical model and, as a consequence, supported their theoretical logic.

Yet, even if postulating the existence and dimensions of these pinholes provided a convenient and convincing experimental explanation, these experiments could not offer a certitude. Fortunately, in the same year, Penner and Martin published an amazing experimental paper reporting the voltammetric behavior of nano-disk arrays. These were nanofabricated by electrochemical deposition of platinum in nanopores created in commercial irradiated insulating polymer membranes (Nuclepore ${ }^{\mathrm{TM}}$ polycarbonate membranes). ${ }^{[11]}$ The size and mean separation of the track-etched nanopores, hence those of the nanodisk electrodes, and their statistical distribution could be fully characterized by electron microscopy, so their existence and characteristics were unquestionable. Therefore, the fact that their voltammetric behavior strictly obeyed the whole set of Amatore and Saveant's theoretical predictions including the trend of voltammetric waves to evolve from textbook$\mathrm{CV}$ ones at slow scan rates towards distorted ones due to steady-state components. ${ }^{[1,12]}$ Moreover, their nanometric dimensions inferred from the theory based on the analysis of their voltammetric behavior were compatible with those observed by electron microscopy.

These three works have been instrumental in installing Amatore and Savéant's theory primacy and experimental usefulness. Owing to the increasing use of electrochemical arrays for a wide variety of physical or (bio)analytical purposes they have been followed by more than 900 papers which took 
advantage of the theory to scrutinize their voltammetric results or derive information. However, except for a few rare ones, ${ }^{[13]}$ no extreme experimental measures were taken to produce regular arrays of nano-disks or band electrodes as those considered in the theory. This is even more true when the arrays consist of statistical dispersion of electrocatalytic nano-objects on conductive surfaces. ${ }^{[14-}$ 17] Thus, one rather relies on the implicit assumption that Amatore and Saveant's theoretical predictions will hold, and surprisingly, this seems to work adequately.

This is, evidently, gratifying for the theory but is nonetheless highly astonishing! Indeed, the peculiar voltammetric behavior of any regular array of nano-active objects stems from the fact that as soon as the extent, $\delta \sim(\pi D R T / F v)^{1 / 2}$, of the diffusion layers generated by the individual activities of each of these objects becomes much larger than their mean separation distance, $d$, they collapse and merge together to generate a collective planar diffusion wave progressing towards the solution bulk, ${ }^{[1,12,18]}$ as it has been established experimentally later by optical and ECL methods, including for application to electronucleation of nanoparticles. ${ }^{[19-27]}$ Hence, each given regular array is intrinsically associated to a characteristic transition scan rate $v_{\text {trans }}=(\pi D R T / F) / d^{2}$, i.e., a transition time $t_{\text {trans }}=d^{2} / \pi D$, around which the array shifts from an apparent behavior akin to a classical voltammetric one for planar electrode of identical surface area as that of the whole array $\left(v_{\text {trans }}<v\right)$ towards that featuring the simple addition of the individual steady (disk-type arrays) or quasi-steady state (bandtype arrays) currents generated by each active object as if it was performing alone $\left(v_{\text {trans }}>v\right) \cdot{ }^{[1,12 \text {, }}$ ${ }^{28]}$ Since truly random arrays display a wide range of separation distances values, $d$, they necessarily exhibit a correspondingly even wider range of $v_{\text {trans }}$ values (note that $v_{\text {trans }} \propto d^{-2}$ ) and, accordingly, should not exhibit any identifiable transition limit. ${ }^{[28]}$

This theoretical riddle had to remain a complete mystery during ca. 25 years, that is, until the moment when sufficiently powerful computer facilities became affordable to be used in electroanalysis laboratories. Compton and his group could then publish a series of brilliant articles ${ }^{[29,30]}$ aiming to revisit the central concepts of Amatore and Saveant's theory by redesigning the initial modeling of the voltammetric behavior of individual sites based on collections of cylindrical unit cells ${ }^{[1]}$ whose circular bases surface area were now defined using Voronoi tessellation to fit the random distributions of the electroactive sites on the basal array surface.

This prompted some of us to investigate in full theoretical details the behavior of random arrays of nanodisk in the context of chronoamperometry using 3D-Brownian methods and the same Voronoibased approach introduced by Compton et al. in the context of electroanalytical and electrodeposition problems ${ }^{[28,31-33]}$ Regarding the riddle above, this unfortunately confirmed that the randomness of any array does indeed manifest itself in its chronoamperometric behavior. The relationship between the randomness of an electrochemical array and its chronoamperometric behavior was so indisputable that we were able to demonstrate theoretically that a careful study of the chronoamperometric responses of a random array over a wide range of timescale could make it possible to reconstruct the distribution of its electroactive sites with an excellent precision. ${ }^{[31,32]}$ However, it has been noted ${ }^{[28]}$ that under chronoamperometric conditions performed on the plateau of a redox wave, the surface concentration is imposed at a zero value on each active electrode. Therefore, there is a fundamental difference between amperometric and voltammetric boundary conditions since in the latter electrochemical method the local surface concentration is finely coupled to the concentration flux at the same point all over a voltammetric wave except when Cottrellian decay current range is reached. This led to the postulation that such a fine coupling could in fact mitigate the consequence of randomness so that an array appears more regular than the geometric distribution of its electroactive sites on its surface suggests. This would be because an electroactive disk operating in a small unit cell would consume the material initially contained in its Voronoi-allocated volume much faster than 
would another one located in a larger adjacent unit cell. Therefore, fine local coupling between surface concentrations and concentration fluxes could somehow lead to virtual homogenization of unit cell volumes even when their Voronoi-defined ones differ considerably. To test this idea, the chronoamperometry of a very slow redox system was simulated on a random array of electroactive nano disks in order to ensure a slight coupling. Compared to the results of simulations of the same array performing at the step potential value on a Nernstian redox system, it was then observed that the chronoamperometric behavior evolved somewhat towards that of a uniform array. ${ }^{[34]}$ Specifically, it has then been established that the electrochemical activity of individual sites inferred from Voronoibased approaches results blatantly wrong as soon as the time scale is large enough to allow the diffusion layers generated by each electrode activity to begin to overlap significantly. However, for these chronoamperometric conditions, the ensuing errors on the individual currents almost compensate each other so the accuracy of the overall array current responses are only slightly affected when using a Voronoi tessellation approach as suggested by Compton et al. ${ }^{[29,30]}$

Representative elementary cells:

(a)

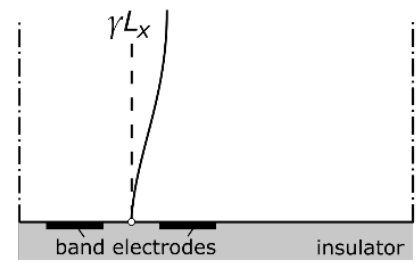

(c)

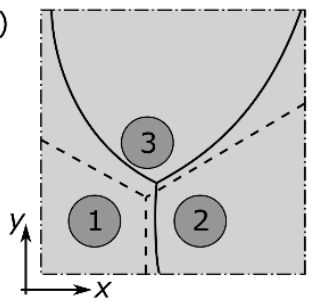

Splitting of the elementary cells into independent Voronoi unit cells

(b)

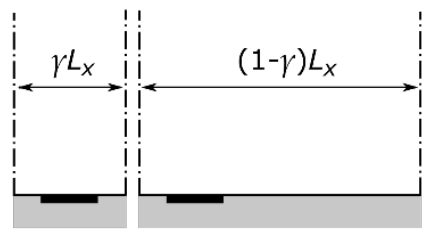

(d)

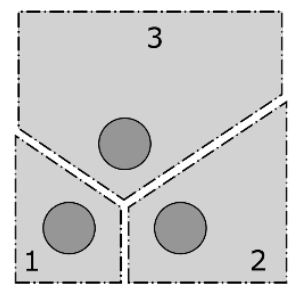

(e)

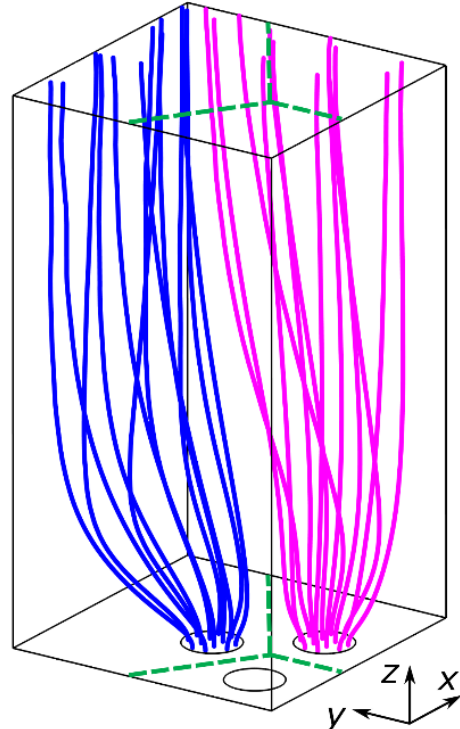

Figure 1. Schematic representation of the systems consisting of band-like and disk-like electrodes/active sites. $a, b)$ side view of the bands system, the size of the system in direction perpendicular to the plane of the figure is much larger than the size of the elementary cell. $c, d)$ top view of the disk system. In a), c) and e) dashed lines represent the boundaries of Voronoi unit cells, while the solid curves feature those resulting from the diffusional competition between the electrodes in chronoamperometry as a function of the distance from the basal plane at infinite time (a) or after an infinite potential step duration at a distance from the array sufficiently large for the bulk solution conditions to be met with (c). ${ }^{[34]}$ Dash-dot lines represent symmetry or no-flux boundaries. $\gamma$ in $(a, b)$ defines the relative sizes $(0<\gamma<1)$ of the Voronoi cells with respect to the width $L_{x}$ of the elementary cell. e) Illustration of the flux line competition between unit cells 1 and 3 in (c,d) as a function of the distance ( $z)$ from the array surface.

In this work, we would like to model the voltammetric behavior of the same systems considered in our previous work ${ }^{[34]}$ with the purpose to examine if the trend observed under chronoamperometric conditions reveals itself with more impact under voltammetric ones. We thus use again the two archetypical pseudo-random arrays consisting of few band-like or disk-like active sites asymmetrically located within an elementary cell (Fig.1) that is periodically repeated to infinity through mirror reflections across each of its vertical faces. ${ }^{[34]}$ This will allow us to examine in detail the specific case 
of voltammetric behaviors of random arrays. For simplicity, in the following we consider a single electron transfer (ET) mechanism in which local surface concentrations and local fluxes are governed by a classical Butler-Volmer relationship. This will suffice to illustrate the problems related to the prediction and analysis of voltammetric results at random arrays.

In the following the analyses will be mostly focused on the long-time behavior of the investigated systems (representative elementary cells of band or disk arrays). ${ }^{[34]}$ Indeed, at shorter times each site tends to behave on its own without interfering with the nearby ones. Moreover, these long-time regimes are those in which the use of arrays is really beneficial. ${ }^{[12]}$ For this reason, owing to the micrometric dimensions of the arrays investigated hereafter, the following simulations were performed using a sufficiently small scan rate value $\left(v=10^{-3} \mathrm{~V} / \mathrm{s}\right)$ to allow full overlap of individual sites' diffusion layers.

\section{Computational details}

The following parameters were used throughout the manuscript: bulk concentration $[A]_{\text {bulk }}=$ $10^{-6} \mathrm{~mol} / \mathrm{cm}^{3}$; diffusion coefficient $D=10^{-5} \mathrm{~cm}^{2} / \mathrm{s}$; transfer coefficient $\alpha=0.5$; formal potential $E_{0}=0 \mathrm{~V}$; band widths $w=10 \mu \mathrm{m}$; band length $L_{z}=1 \mathrm{~mm}$; band elementary cell size $L_{x}=70 \mu \mathrm{m}$; disk radius $r_{d}=10 \mu \mathrm{m}$; disks elementary cell side $L_{x}=L_{y}=100 \mu \mathrm{m}$.

All simulated results reported in the following were obtained using Comsol as described in details in ref.[34] (boundary conditions, mesh settings) with the only difference that the electroactive sites potential was a triangular function of time with a scan rate as indicated in the captions of Figures and Table 1. Note that these geometric parameters values have been selected in the micrometric range to ease the writing of boundary conditions in the simulations since Comsol has to be programed in dimensioned quantities rather than in dimensionless ones. However, provided that all geometric parameters are simultaneously down-scaled by the same factor and the scan rate up-scaled by the squared power of the same factor all reported behaviors will remain identical except for the current amplitudes that will be down-scaled accordingly (see zone diagram and discussion below).

\section{Results and discussion}

\section{Band array}

The voltammetric behavior of the elementary cell of a band pseudo-random array shown in Fig.1a,b ${ }^{[34]}$ was assessed under fast and slow electron transfer (ET) conditions assuming a Butler-Volmer relationship between the local surface concentration of the substrate $A$ initially present in the bulk at concentration $[A]_{b u l k}$ and its flux at the same point, viz.,:

$$
D \frac{\partial[A]}{\partial \vec{n}}=k_{0} \exp \left(\frac{(1-\alpha) F}{R T}\left(E-E_{0}\right)\right)[A]-k_{0} \exp \left(-\frac{\alpha F}{R T}\left(E-E_{0}\right)\right)\left([A]_{b u l k}-[A]\right)
$$

where $D$ is the diffusion coefficient assumed to be equal for species $A$ and its product; $k_{0}$ is the standard heterogeneous rate constant; $E_{0}$ is the formal potential of the $A / B$ redox couple; $E$ is the electrode potential; $\vec{n}$ is the unity vector normal to the surface at the local point where Eqn. (1) applies.

The total currents simulated for the whole elementary cell upon letting each band to freely interact (i.e., allowing a free competition between their individual diffusion layers) are in a close agreement with those obtained through summation of the two individual currents generated in the physically 
separated Voronoi cells (see Fig.2). A small difference localized around the two CV peaks amounts to less than $1 \%$ or $3 \%$ under Nernstian or slow charge transfer, respectively, but the two CVs are almost superimposed over the whole scan. This emphasizes the great conceptual utility of the Voronoi tessellation as well as its efficiency for the evaluation of the total array currents.

However, as in the case of amperometry ${ }^{[34]}$, the simulated currents flowing at each electrode (i.e., for each unit cells) highly differed depending whether they were evaluated by deducing their individual values from whole cell simulations or directly obtained using the Voronoi approximation as evidenced in Fig.3.
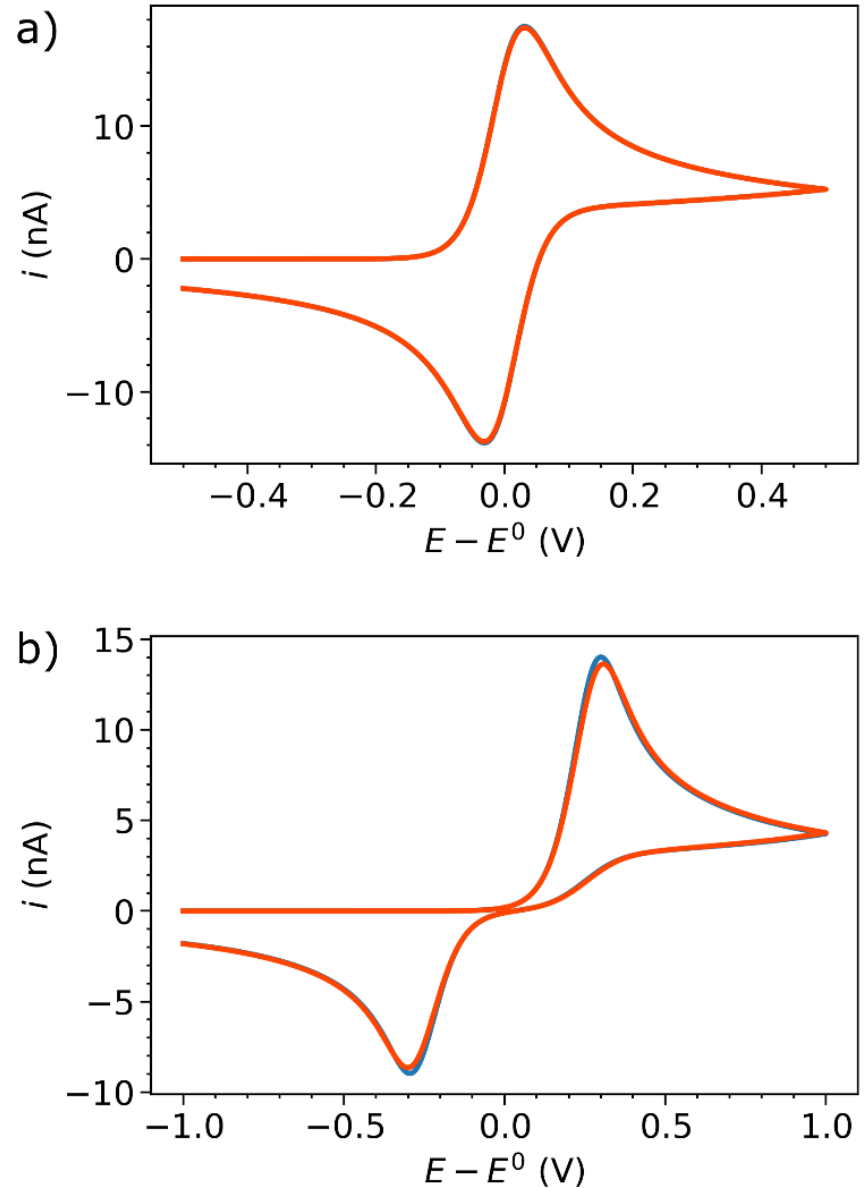

Figure 2. Simulated total currents for the bands system shown in Fig.1a, $b$ obtained for the whole elementary cell (blue) or for Voronoi cells (orange) obtained at $v=10^{-3} \mathrm{~V} / \mathrm{s}$ and a) $\left.k_{0}=10^{2} \mathrm{~cm} / \mathrm{s} ; \mathrm{b}\right) k_{0}=10^{-5} \mathrm{~cm} / \mathrm{s}$. All other parameters were as given at the end of introduction.

For example, Fig.3a compares the electrode-wise CVs for the case of Nernstian kinetics. It is seen that the current peak amplitudes of the individual CVs predicted for the left and right electrode through each method drastically differ. Moreover, although under a Nernstian regime one does not expect any potential shift, one notices that the peak potential values obtained through the Voronoi method for each band differ by a ca. $5 \mathrm{mV}$. By comparison, those deduced from the whole elementary cell simulation result almost identical, in line with the fact that the peak current values are also more close than the ones obtained for each Voronoi unit cell. For the Voronoi unit cells, the individual current 
peaks amplitudes are expected to feature precisely their geometrical surface area (Fig.1b and Table 1), which is by construction the same at any height from the electrode plane. However, in each Voronoi unit cell, the exact time range (i.e. the range of potential) over which the transition from the quasicylindrical diffusion regime that prevails near the electrodes' plane to the planar diffusion regime which predominates deeper in the solution is a function of the shape of the unit cell and of its size vs. that of the active electrode. ${ }^{[1,12,32,34]}$ Hence, the two individual $i(E)$ functions do not vary exactly in phase for each Voronoi unit cell thus leading to a slight difference in waves shapes, the CVs being not exactly homothetical. As a consequence, their relative current amplitudes taken at their peak potentials are very close to those of the Voronoi unit cells surface areas but without being exactly equal as can be seen in the corresponding column of Table 1.
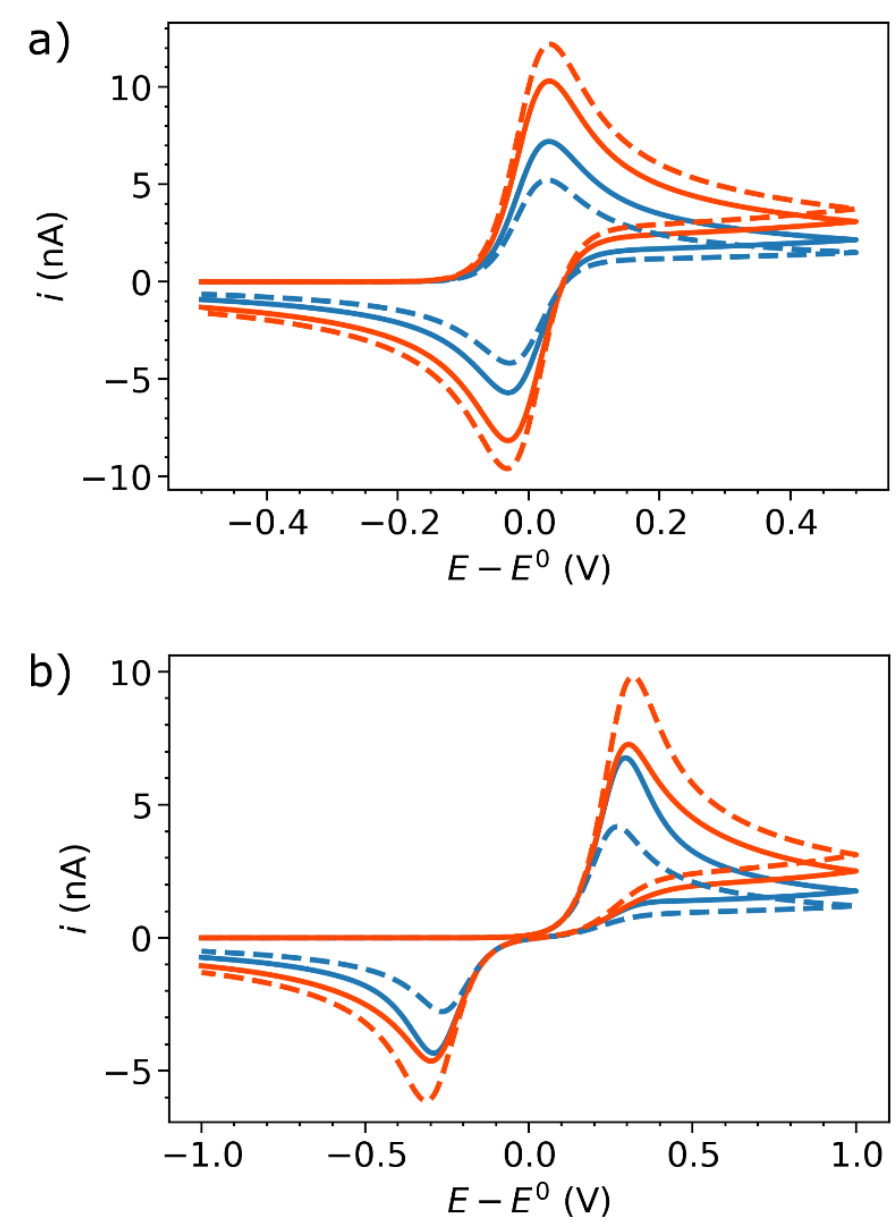

Figure 3. CVs simulated for individual bands at the whole elementary cell or with Voronoi unit cells shown in Fig.1a,b for a) $k_{0}=10^{2} \mathrm{~cm} / \mathrm{s}$ and b) $k_{0}=$ $10^{-5} \mathrm{~cm} / \mathrm{s}$, and $v=10^{-3} \mathrm{~V} / \mathrm{s}$. The individual voltammetric currents are shown in solid curves when they are deduced from whole elementary cell simulations or dashed ones when simulated independently for each Voronoi unit cell for each electrode (blue: left band electrode; orange: right band one). All other parameters were as given at the end of introduction.

In the case of the whole elementary cell simulations the unit cells can be defined by considering the bundles of flux lines originating from each electrode ${ }^{[34]}$ (see e.g. visualization in Fig.1e for the case of disk-like active sites). The cross-section surface area values of each unit cell defined by the flux lines bundles become more and more close upon increasing the distance from the array basal plane in 
which the electrodes are embedded (Fig.1a and Table 1). Indeed, at $z=0$ the surface areas of both unit cells are identical to those of the corresponding Voronoi cells (Fig.1a). Conversely, while the diffusion layers of individual electrodes propagate towards the solution, the outcome of their increasing competitive interactions causes the bundle of flux lines associated to the left band (that with the smallest Voronoi cross-section) to expand laterally and compress that originating from the right band, as outlined in Figure 1a by the shape of the boundary (solid curve) separating the two individual diffusion layers. Owing to the law of flux conservation, each current amplitude is necessarily proportional to the cross-section area reached by its flux line bundle at the distance from the array basal plane where the bulk solution conditions are reached. The amplitudes of the CVs obtained with whole cell simulations follow this relation (Fig.3a and Table 1) and tend to equalize.

Table 1. Voronoi cells surface area $A_{\text {cell }}^{\text {Voronoi }}$ and values of the cross-section areas $A_{\text {cross }}$ of the bundles of flux lines responsible for determining individual currents monitored at each active site at the end of the diffusion layers under the conditions indicated in column captions as deduced from the individual current intensities (see text). All values are given in percentages relative to the global area of each elementary cell accordingly.

\begin{tabular}{|c|c|c|c|c|c|c|}
\hline & \multirow[b]{2}{*}{$\begin{array}{l}\text { Unit } \\
\text { Cell } \\
\text { (see } \\
\text { Fig.1) }\end{array}$} & \multirow[b]{2}{*}{$\begin{array}{l}A_{\text {cell }}^{\text {Voronoi }}(\%) \\
\text { (Voronoi } \\
\text { areas) }\end{array}$} & \multirow{2}{*}{$\begin{array}{c}A_{\text {cross }}(\%) \\
\text { based on } \\
\text { long-time } \\
\text { limit of } \\
\text { amperometric } \\
\text { currents in } \\
\text { whole cell } \\
\text { simulations }\end{array}$} & \multirow{2}{*}{$\begin{array}{c}A_{\text {cross }}(\%) \\
\text { based on } \\
\text { Voronoi CV } \\
\text { simulations }{ }^{(b, c)} \\
\\
\text { Based on } \\
\text { currents peak } \\
\text { ratios }\end{array}$} & \multicolumn{2}{|c|}{$\begin{array}{c}A_{\text {cross }}(\%) \text { based on } \\
\text { whole cell CV } \\
\text { simulations }^{(c)}\end{array}$} \\
\hline & & & & & $\begin{array}{l}\text { Based } \\
\text { on } \\
\text { currents } \\
\text { peak } \\
\text { ratios }\end{array}$ & $\begin{array}{c}\text { Based on } \\
\text { currents } \\
\text { ratios at } \\
\text { inversion } \\
\text { potential } \\
E_{\text {inv }}\end{array}$ \\
\hline \multicolumn{7}{|c|}{ Band array (Fig.1a,b) } \\
\hline \multirow{2}{*}{$k_{0}=10^{2} \mathrm{~cm} / \mathrm{s}$} & left & 29 & 41 & 30 & 41 & 41 \\
\hline & right & 71 & 59 & 70 & 59 & 59 \\
\hline \multirow{2}{*}{$k_{0}=10^{-5} \mathrm{~cm} / \mathrm{s}$} & left & 29 & 48 & 30 & 48 & 41 \\
\hline & right & 71 & 52 & 70 & 52 & 59 \\
\hline \multicolumn{7}{|l|}{ Disk array (Fig.1c,d) } \\
\hline \multirow{3}{*}{$k_{0}=10^{2} \mathrm{~cm} / \mathrm{s}$} & 1 & 17 & 29 & 19 & 29 & 29 \\
\hline & 2 & 29 & 34 & 31 & 34 & 34 \\
\hline & 3 & 54 & 37 & 50 & 37 & 37 \\
\hline \multirow{3}{*}{$k_{0}=10^{-5} \mathrm{~cm} / \mathrm{s}$} & 1 & 17 & 33 & 19 & 32 & 29 \\
\hline & 2 & 29 & 33 & 31 & 34 & 34 \\
\hline & 3 & 54 & 34 & 51 & 35 & 37 \\
\hline
\end{tabular}

a) Values taken from ref.[34] for simulated anodic chronoamperometric steps performed at $E_{\text {step }}=E_{0}+0.3 \mathrm{~V}$ for each $k_{0}$ values. b) See text for the slight differences between the relative values of $A_{\text {cross }}$ and $A_{\text {cell }}^{\text {Voronoi }}$. c) Based on voltammograms simulated at $v=10^{-3} \mathrm{~V} / \mathrm{s}$, inversion potentials for both band and disk arrays for $k_{0}=10^{2} \mathrm{~cm} / \mathrm{s}$ were $E_{\text {inv }}=E_{0}+0.5 \mathrm{~V}$, while $k_{0}=10^{-5} \mathrm{~cm} / \mathrm{s}$ were $E_{i n v}=E_{0}+1.5 \mathrm{~V}$. All other parameters including arrays geometrical characteristics were as summarized in the Computational details section.

Similar trends, although accentuated, are observed for the slow charge transfer case when the individual currents are deduced from simulation of the whole elementary cell (Fig.3b and Table 1). This confirms that the fact of imposing a strong coupling between local surface concentrations and local fluxes imposes a stronger diffusion competition between the two bands. Hence, the crosssections areas of their flux lines bundles, $A_{\text {cross }}$, at the end of the diffusion layers tend to equalize faster when the simulations are performed in the whole cell. Relative $A_{\text {cross }}$ values were deduced from the current intensities values at each electrode based on a Cottrell-type formulation, viz., $i \propto$ 
$A_{\text {cross }}$. In this respect, it is interesting to remark that while the individual CVs current amplitudes are almost identical before and over their maxima (Fig.3b), their Cottrellian tails tend to diverge to display relative amplitudes similar to those observed in Fig.3a for the Nernstian case. The same is true for the backward waves. This definitively establishes that the diffusional competition between the two electrodes has not an irreversible character but is a dynamic phenomenon, being more intense when the coupling between the local surface concentrations and the local fluxes is the strongest and lessening when this coupling tends to decrease.

Evidently, this cannot be the case for independent simulations of each Voronoi cells, for which the unit cells surface areas, $A_{\text {cell }}^{\text {Voronoi }}$, is determined once for all by the tessellation mode of the basal plane of the array and is not modified along the course of a voltammogram. The relative contributions of individual sites obtained upon considering simulations restricted to each Voronoi cell result, as expected, almost identical to those obtained in the case of fast kinetics (Table 1). In addition, it is noted that the two individual CVs peak potentials are now significantly different in agreement with the prediction that the difference between the surface areas of the electroactive site, $A_{\text {site }}$, and that of the unit cell, $A_{\text {cell }}^{\text {Voronoi }}$, increases the charge transfer slowness as if an apparent rate constant $k_{0}^{a p p}=k_{0} A_{\text {site }} / A_{\text {cell }}^{\text {Voronoi }}$ was involved. ${ }^{[1]}$ Accordingly, the peak-to-peak separation, $\Delta E^{p}=E_{\mathrm{Ox}}^{p}-$ $E_{\mathrm{Red}}^{p}{ }^{[35]}$ is smallest for the $\mathrm{CV}$ simulated in the left Voronoi unit cell for which $k_{0}^{a p p}$ is the largest since

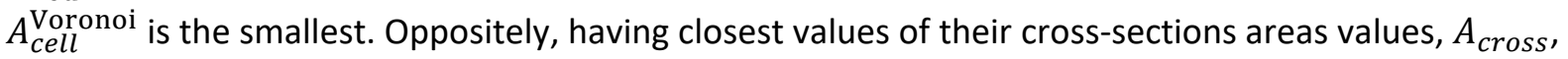
the CVs predicted for each electrode based on simulations of the whole elementary cell correspond to close rate constants values, $k_{0}^{a p p}=k_{0} A_{\text {site }} / A_{\text {cross }}$, i.e., to close $\Delta E^{p}$ values.

It is thus extremely clear that the shape of the individual electrodes CVs depends on the type of the boundary condition (fast or slow kinetics, but also of the kinetic regime) and extent (duration of the experiment) of the dynamic diffusional competition between the neighboring sites. However, albeit the individual CVs are wrongly predicted based on a Voronoi tessellation approach, it must be stressed that, at least for simple electron transfer mechanisms, the global CVs relative to the whole elementary cell are correctly predicted with negligible errors compared to those arising in a practical situation considering the experimental uncertainties on the positions of the electroactive sites of random arrays.

\section{Disk array}

The voltammetric behavior of the pseudo-random array with elementary cell containing three asymmetrically placed disk-like active sites shown in Fig.1c was investigated following the same simulation strategy developed above for the band-electrodes array. Owing to the mean distances values between the disk electrodes and diffusion coefficient (see Computational details section), a slow scan rate of $v=10^{-3} \mathrm{~V} / \mathrm{s}$ was again selected to allow a sufficient overlap of the individual layers and a Cottrell-like behavior of the whole array, ${ }^{[1]}$ and two electron transfer rate constants with sufficiently large differences in magnitudes $\left(k_{0}=10^{2} \mathrm{~cm} / \mathrm{s}\right.$, and $\left.k_{0}=10^{-5} \mathrm{~cm} / \mathrm{s}\right)$ were again selected to investigate and compare the behavior of Nernstian, Fig.4, and slow charge transfer, Fig.5, electrochemical systems.

In accordance with our previous results for amperometry ${ }^{[34]}$ and those reported above for bands array, the total currents obtained in whole cell or Voronoi cells for both fast and slow ET kinetics (Figs. 4a and $5 a$ ) agreed extremely well since they exhibited less than $5 \%$ maximum relative differences between the simulated peak currents. Contrastingly, the individual electrode contributions resulted significantly different, depending drastically on the simulation approach used (viz., considering the 
elementary cell as a whole as in Fig.1c, or separately for each Voronoi unit cell represented in Fig.1d). This is indeed, extremely similar to what was obtained above for the band-electrodes array and occurs due to the rearrangement of the individual diffusion layers discussed above.
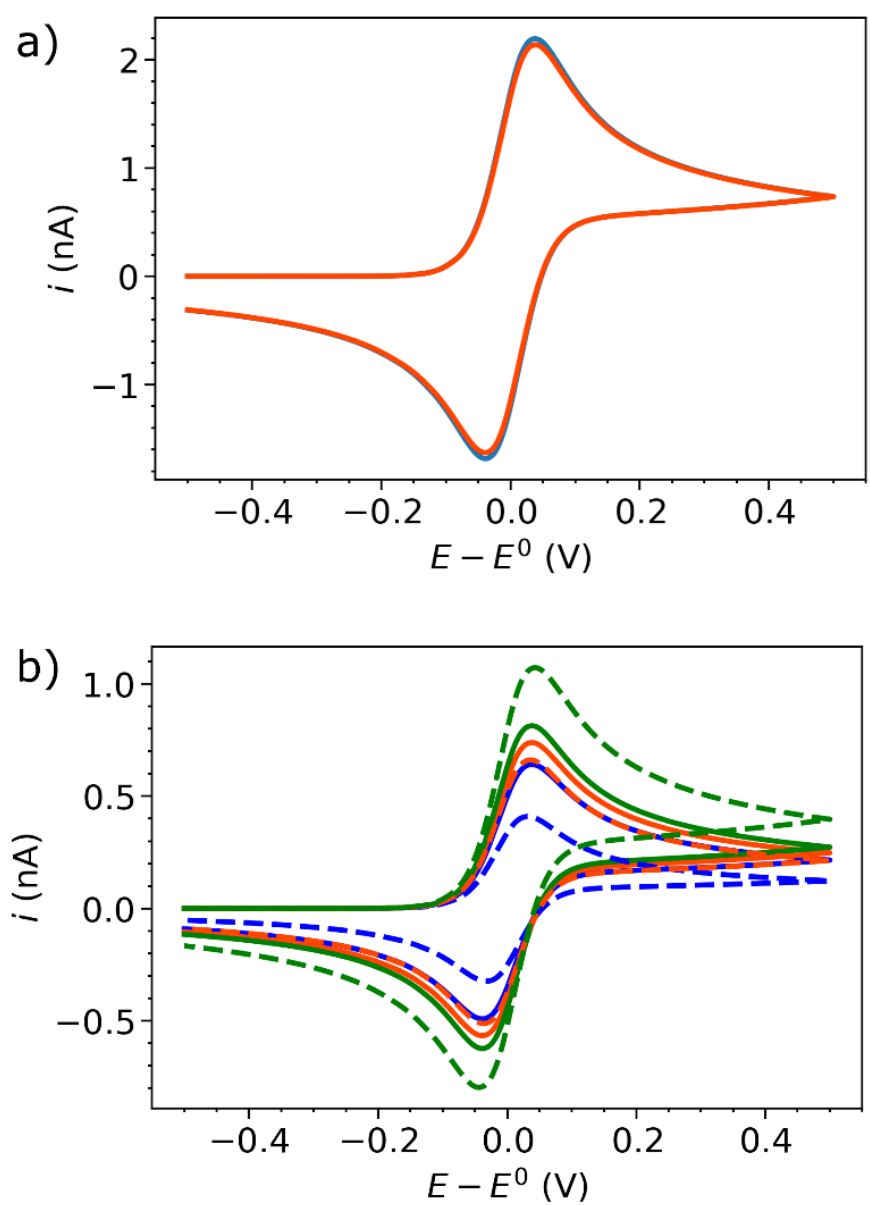

Figure 4. CVs simulated at a scan rate $v=10^{-3} \mathrm{~V} / \mathrm{s}$ for $k_{0}=10^{2} \mathrm{~cm} / \mathrm{s}$. a) total currents (blue whole elementary cell; orange - Voronoi unit cells); b) electrode-wise currents for the Voronoi cells (dashed lines), or deduced from whole elementary cell simulations (solid lines). In b) blue, orange and green lines relate to the currents flowing through electrodes 1, 2 and 3 (Fig.1C,d), respectively. Disks radii are $r_{d}=10 \mu \mathrm{m}$. All other parameters including arrays geometrical characteristics were as summarized in the Computational details section.

This rearrangement of the diffusion layers volumes along the distance from the array basal plane (compare Figure 1e) has a double effect. Firstly, as discussed above, the long-time response of each site is directly proportional to the surface areas, $A_{\text {cross }}$, of the cross-section of the flux lines delimited unit cells taken at large distances from the array basal plane. Actually, as already noted for the bandelectrodes array, owing to the flux conservation within each separate bundle of flux lines, each crosssection surface area directly commands the flux of bulk molecules which is ultimately captured by the disk-electrode that generates this bundle of flux lines. Consequently, the surface areas of these crosssections delimit the fractions of the bulk solution that "feed" each active site at long times in a Cottrelllike planar regime, ${ }^{[1,28,32,34]}$ in opposition to the Voronoi surface areas of each tesselate, $A_{\text {cell }}^{\text {Voronoi, }}$, which defines the amplitude of their short-time response (i.e., before the diffusional patterns of each electrode start to overlap). 

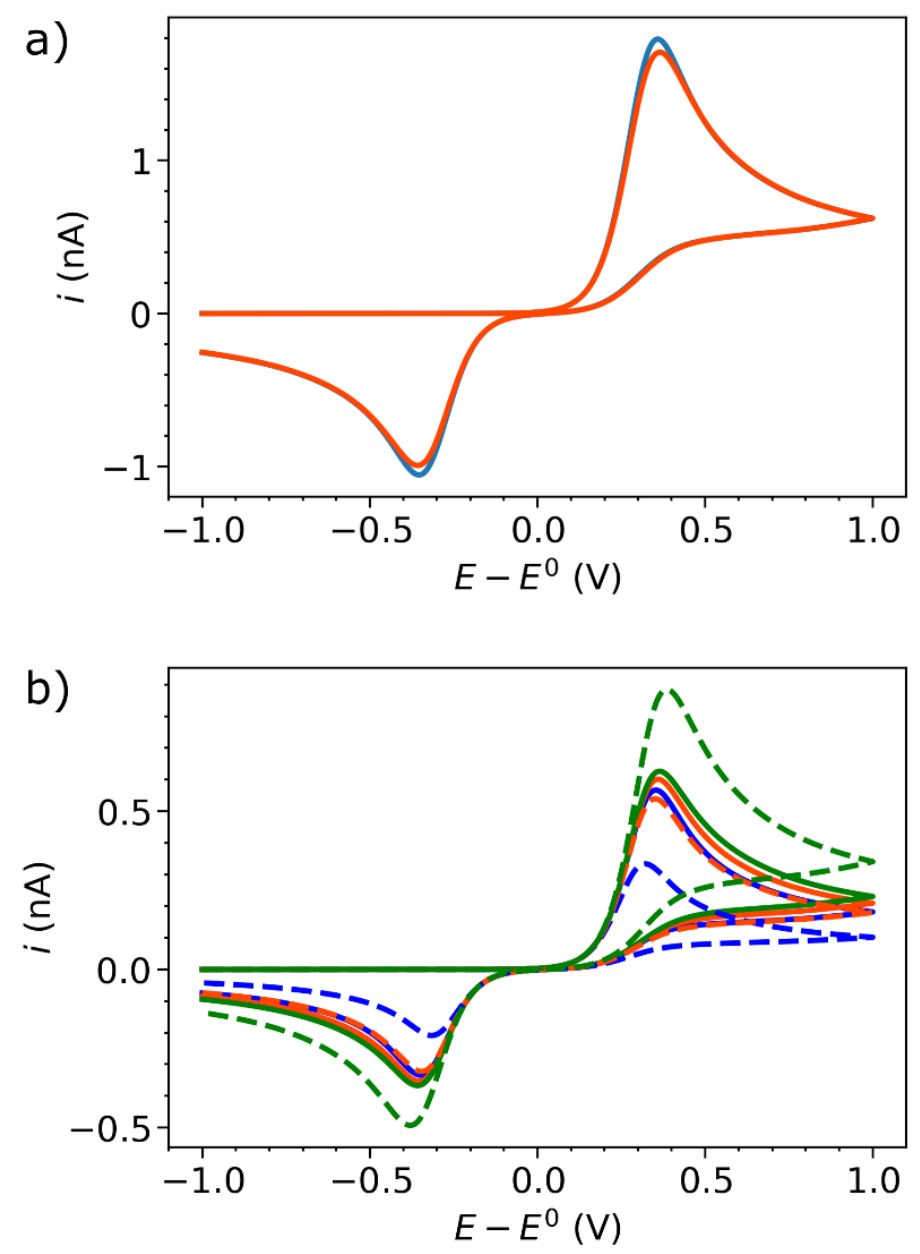

Figure 5. CVs for disk system at $v=10^{-3} \mathrm{~V} / \mathrm{s}$ and $k_{0}=10^{-5} \mathrm{~cm} / \mathrm{s}$. a) total currents (blue-whole elementary cell; orange - Voronoi unit cells); b) electrode-wise currents for Voronoi cells (dash), whole elementary cell (solid). In b) blue, orange and green lines relate to the currents flowing through electrodes 1, 2 and 3 (Fig.1c,d), respectively. Disks radii are $r_{d}=10 \mu \mathrm{m}$. All other parameters including arrays geometrical characteristics were as summarized in the Computational details section.

Secondly, the average relative surface area of the active sites to those, $A_{\text {cross }}$, of their apparent unit cells, i.e., the ratio $\rho^{\infty}=A_{\text {site }} / A_{\text {cross }}$, defines the type of the voltammetric response for a given intrinsic electron transfer rate constant $k_{0}$ after sufficiently long diffusional times. ${ }^{[1]}$ As shown in Table 1 , and already rationalized above for the two-band elementary cell, the cross-section surface areas $A_{\text {cross }}$ of the bundles of flux lines originating from each active disk electrode tend to become rather similar far away from the array basal plane. This trend is the most effective when the coupling between the local surface concentrations and local fluxes has the strongest influence on the concentration value, i.e., for a slow charge transfer and around the peaks of the forward and backward CV waves. This is readily apparent through observing the three almost identical CVs deduced for each disk from whole cell simulations for the slow ET in Fig.5b $\left(k_{0}=10^{-5} \mathrm{~cm} / \mathrm{s}\right)$ to the Nernstian ones in Fig. $4 \mathrm{~b}$ that are still visibly different although more similar than those predicted upon considering a Voronoi tessellation approach whatever the electron transfer rate constant (see Figs. $4 \mathrm{~b}$ and $5 \mathrm{~b}$ ). Conversely, evaluating the relative $A_{\text {cross }}$ values from the relative current values in the very Cottrellian ranges of the CVs, i.e., at the inversion point of the potential scan (Table 1, last column) where the surface substrate concentration is the same for all electrodes, the electrochemical boundary conditions are 
identical for each individual unit cell so that the relative $A_{\text {cross }}$ values result identical for both fast and slow kinetics.

Even so, the variance associated to the distributions of $\rho^{\infty}$ values in each case is considerably smaller than that of the Voronoi corresponding values, $\rho^{\text {Voronoi }}=A_{\text {site }} / A_{\text {cell }}^{\text {Voronoi }}$, featuring each individual tessellated cells. ${ }^{[28,32,34]}$ This explains a posteriori the puzzling observation that modelling the voltammetric behavior of random arrays based on a theory originally designed for regular arrays of identical active nanometric sites ${ }^{[1]}$ provides much better outcomes than one would have ever guessed taking into account the large dispersion of the surface areas of the unit cells defined through considering the distribution of the electroactive sites on the basal array plane ${ }^{[8,11,13]}$.

To continue on this point, we must first note that the slow scan rate regimes referred to in the discussion above cannot be defined in absolute terms. Indeed, on the one hand this concept implies that the scan rate is sufficiently low for the thickness of the layer in which the solution composition is altered vs. the bulk one largely exceeds the mean inter-sites distance in order to allow a total competitive adjustment of the flux lines generated by each electroactive site. ${ }^{[1,34]}$ If this would not be the case classical-looking voltammetric waves could not be observed since the voltammograms would be corrupted by strong components of steady state diffusion. ${ }^{[1,36]}$ On the other hand, would the scan rate be too small, based on usual expectations in voltammetric analyses, ${ }^{[6]}$ one would expect that voltammetric waves always exhibit characteristics close to Nernstian (viz. as in Figure 4) and that no quasi-reversible or irreversible behavior similar to those shown in Figure 5 could to be observed. In practice, this does not happen because under the present conditions, for any given $k_{0}$ value, the degree of reversibility is not commanded by the scan rate alone as in usual voltammetry ${ }^{[6]}$ but also by the huge curvatures of the flux lines near each electroactive site. ${ }^{[1,34]}$ Indeed, these curvatures enforce much larger local diffusion rates, $D / r_{d}^{2}$, where, as defined above, $r_{d}$ is the common electroactive site radius and $D$ is the common diffusion coefficient of electroactive species, ${ }^{[36]}$ than that, $F v / R T,{ }^{[6,7]}$ which depends on the scan rate. Note that the value of $\theta^{\mathrm{CV}}=R T / F v$ defines the time scale that commands the extent of the diffusion layer propagation towards the solution bulk in a voltammetric experiment.

In fact, the solution of this conundrum has been published ca. 40 years ago for regular hexagonal disks arrays and for bands arrays. ${ }^{[1]}$ The voltammetric behavior of these systems was established to depend on two main dimensionless parameters, viz., a kinetic one characterizing the effect of the intrinsic rate constant $k_{0}$ on the degree of the electron transfer irreversibility of the voltammetric wave (viz., Nernstian-like vs irreversible ET) under the present conditions:

$$
\Lambda^{\infty}=k_{0} \rho^{\infty} \sqrt{\frac{R T}{D F v}}
$$

and a second one (for disks arrays):

$$
\zeta^{\infty}=\frac{\rho^{\infty}}{0.6} \sqrt{\left(\frac{D}{r_{d}^{2}}\right) /\left(\frac{F v}{R T}\right)}
$$

which compares the magnitudes of the two diffusion rates introduced above since their relative values control the overall effect of diffusional transport on the voltammetric behavior, viz the wave shape from near-classical peak-shaped ones (as in Figures $4 \& 5$ ) to steady state sigmoid ones. $\Lambda^{\infty}$, the dimensionless electron transfer rate constant in Eqn. (2), has a structure similar to that, $\Lambda^{\text {planar }}=$ $k_{0}(R T / D F v)^{1 / 2}$, applying at fully electroactive planar electrodes, ${ }^{[6]}$ but evidences that for arrays the intrinsic ET rate constant $k_{0}$ is replaced by a diminished apparent one, $k_{0}^{\text {app }}=k_{0} \rho^{\infty}$, in which the factor $\rho^{\infty}=A_{\text {site }} / A_{\text {cross }}$ accounts for the strong rates of diffusion imposed by the large flux lines 
curvatures near the disk electrodes. The smallest is the size of the active component vs. the insulating one, the smallest is $\rho^{\infty}$ and the smallest $k_{0}^{\text {app }}$. The effects of these two parameters can conveniently be visualized under the form of a zone diagram such as that represented in Figure 6 adapted from ref.[1].

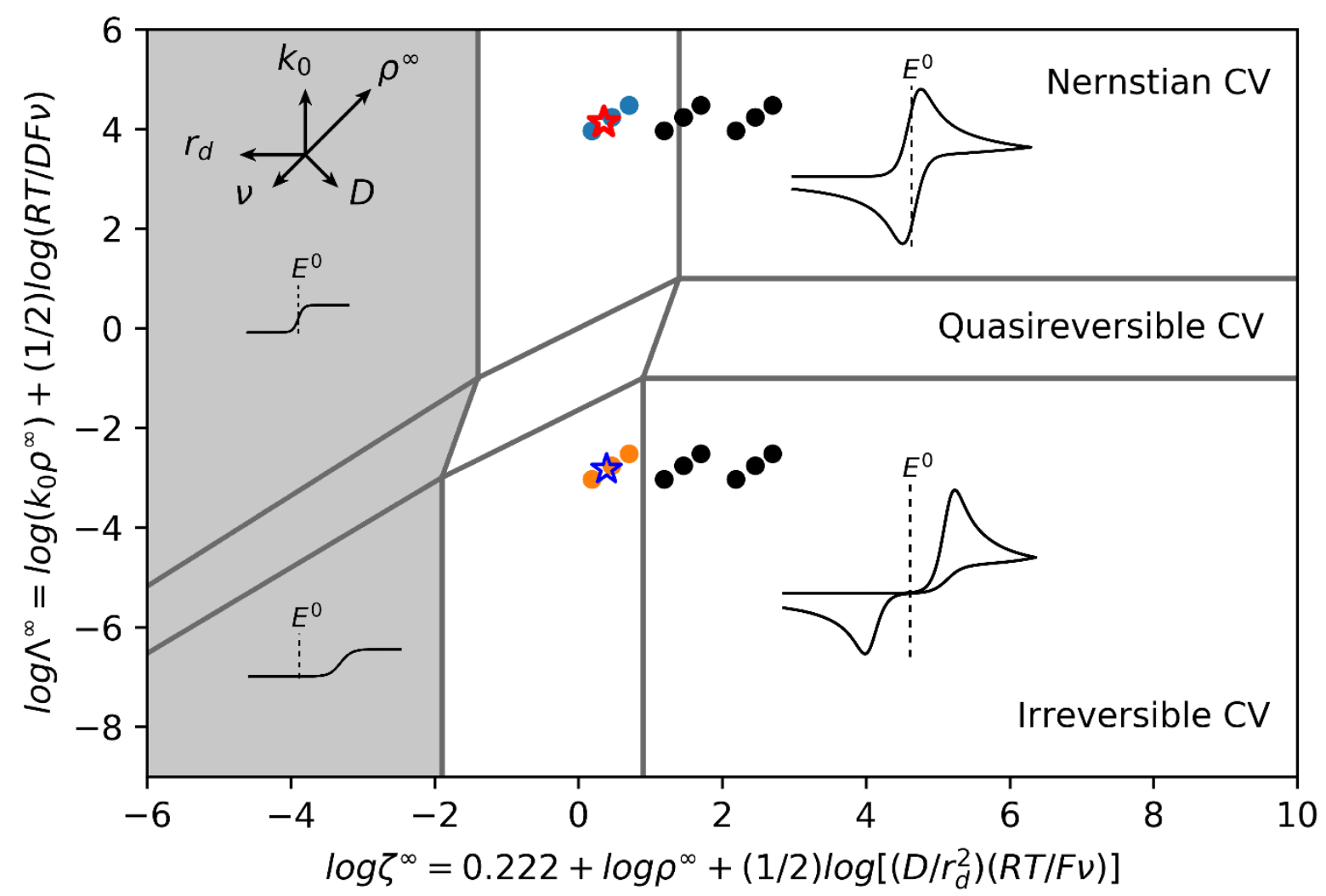

Figure 6. Zone diagram adapted from that in ref.[1] (the zones shown in grey shade correspond to steady state sigmoid voltammograms since they are irrelevant to our purposes in this work because they correspond to too small $\log \zeta^{\infty}$ values to allow any significant interactions between the diffusion layers generated by each active site; note also that another set of zones also irrelevant to our purpose here develop on the left of the grey shaded zones featuring very small classical CVs when $\log \zeta^{\infty}$ values becomes sufficiently even smaller to allow planar diffusion layers to develop over each disk electrode, see refs. $[1,12,28])$. The locations of the three individual Voronoi cells shown in Fig.1c are shown by filled circular symbols (blue: $k_{0}=10^{2} \mathrm{~cm} / \mathrm{s}$; yellow: $k=10^{-5} \mathrm{~cm} / \mathrm{s}$ ), based on their different $\rho^{\text {Voronoi }}=$ $A_{\text {site }} / A_{\text {cell }}^{\text {Voronoi }}$ values instead of $\rho^{\infty}$. The red $\left(k_{0}=10^{2} \mathrm{~cm} / \mathrm{s}\right)$ and blue $\left(k=10^{-5} \mathrm{~cm} / \mathrm{s}\right)$ empty star symbols show almost identical locations of the three cells whose close $A_{\text {cross }}$ values, hence their close $\rho^{\infty}=A_{\text {site }} / A_{\text {cross }}$ values, are deduced from the tracking of their flux lines bundles as they emerge in whole cells simulations (see text). $v=10^{-3} \mathrm{~V} / \mathrm{s} ; D=10^{-5} \mathrm{~cm}^{2} / \mathrm{s}$; transfer coefficient $\alpha=0.5$; active disks radii $r_{d}=10 \mu \mathrm{m}$; elementary cell side $L_{x}=L_{y}=100 \mu \mathrm{m}$. The four triplets of solid black symbols correspond to the locations of the same three Voronoi unit cells after their geometrical dimensions are down-scaled by a factor 10 or 100 (see text). The compass in the top left corner of the zone diagram illustrates the effect on the system position of an increase by a factor 10 of each parameter indicated.

The boundaries shown in Fig. 6 were determined in ref. [1] for regular arrays of disk electroactive sites arranged on a periodical hexagonal lattice, and modeled by cylindrical cells (see next section) so they correspond to a single set of $A_{\text {cell }}^{\text {Voronoi }}=A_{\text {cross }}$ and $\rho=A_{\text {site }} / A_{\text {cell }}^{\text {Voronoi }}=A_{\text {site }} / A_{\text {cross }}$ values. However, for the array shown in Fig.1c, each of the three unit cells has to be associated to two 
different sets of $\rho$ values. One, $\rho^{\infty}$, which has been introduced above and is deduced from simulations of the whole elementary cell provides almost identical values within a few percent for all three unit cells since their $A_{\text {cross }}$ values are extremely similar (Table 1 ). The locations of the system in the diagram may then be featured by the empty star symbols depending on the values of the rate constants $k_{0}$, hence of $\log \Lambda^{\infty}$. The other one, is defined according to the Voronoi tessellation concept as in refs. $[28,32,34]$ and affords three rather different $\rho^{\text {Voronoi }}=A_{\text {site }} / A_{\text {cell }}^{\text {Voronoi }}$ values (Table 1 ). Accordingly, each of the three unit cells is represented by one colored filled circle for each rate constants $k_{0}$ (note that the larger the unit cell, the smaller $\Lambda^{\infty}$ and $\zeta^{\infty}$ in the group of three representative symbols).
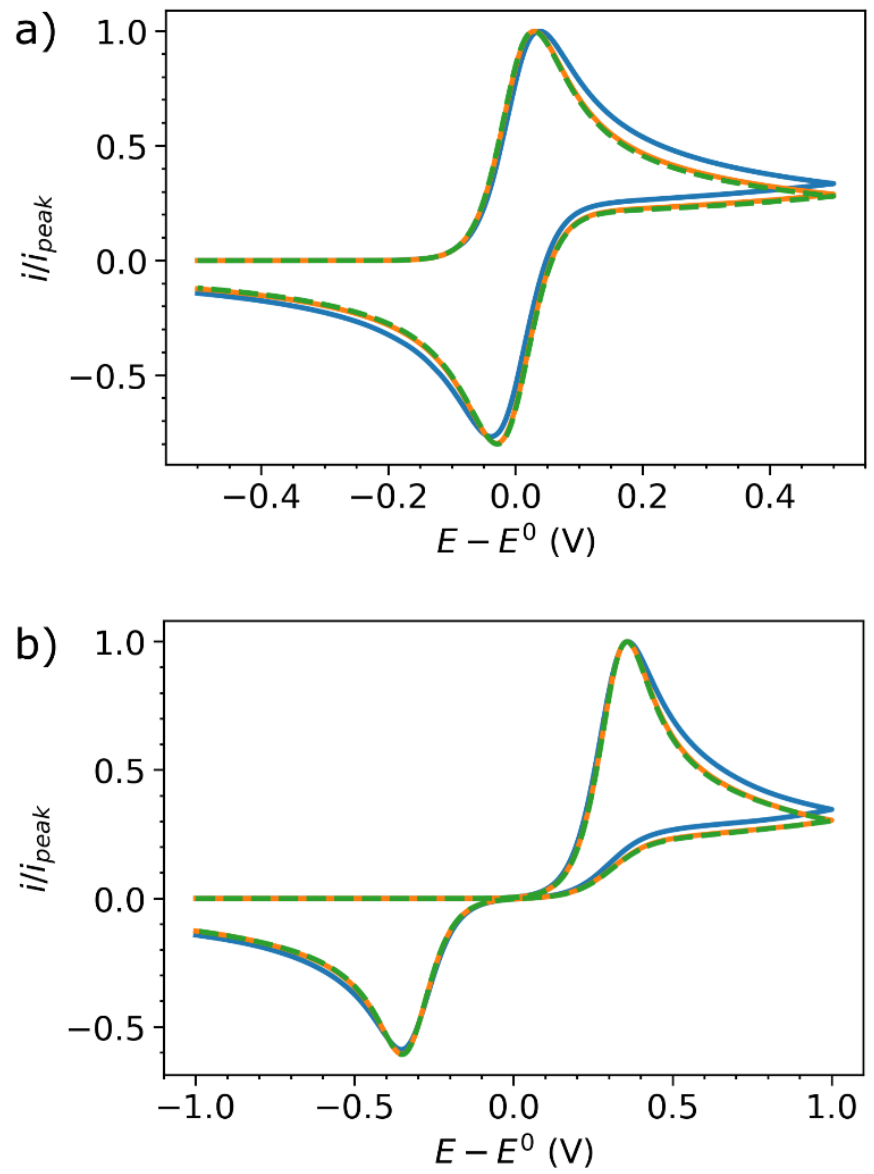

Figure 7. Total CV current (normalized by forward peak current value) simulated at a whole elementary cell for three disk system at $v=10^{-3} \mathrm{~V} / \mathrm{s}$, a) $k_{0}=10^{2} \mathrm{~cm} / \mathrm{s}$ and b) $k_{0}=$ $10^{-5} \mathrm{~cm} / \mathrm{s}$. Blue $\mathrm{CV}$ : disk radii $r_{d}=10 \mu \mathrm{m}$ and total surface area of the elementary cell $A_{\text {tot }}=10^{-10} \mathrm{~m}^{2} ;$ yellow CV: $r_{d}=1 \mu \mathrm{m}$ and $A_{\text {tot }}=10^{-12} \mathrm{~m}^{2} ;$ green CV: $r_{d}=100 \mathrm{~nm}$ and $A_{\text {tot }}=10^{-14} \mathrm{~m}^{2}$ (note that it is shown in dashed line to allow the comparison with the almost identical yellow (V). All other parameters including arrays geometrical characteristics were as summarized in the Computational details section. See the last paragraph of the Cylindrical approximation of unit cells section for further details about this set of CV simulations.

Note that two groups of triplets of black solid circles are shown in addition for each $k_{0}$ value in order to illustrate the effect of a homothetic down-scaling of the elementary cell dimensions by a factor 10 
or 100 , i.e., corresponding to an increase of $\log \zeta^{\infty}$ by one or two units respectively. Indeed, for the dimensions and conditions considered in this work (see Fig.6 caption and the Computational details section), one notices that the points featuring the systems at hand are not completely located into the classical Nernstian or irreversible domain ${ }^{[6]}$, but still occupy part of the transition zones. This implies that the corresponding voltammograms cannot display fully canonical shapes but are partially "corrupted" by the involvement of edge effects. ${ }^{[1,12,28]}$ In order to quantify the effect of the corresponding distortions the same systems were simulated assuming that all their geometrical dimensions were down-scaled by a factor 10 or 100 while every other parameter was kept the same (note that down-scaling all geometrical dimensions does not affect the $\rho$ values). Fig.7 present the outcome of this 10 or 100 down-scaling on the global voltammograms simulated within the elementary cells as a whole for each $k_{0}$ value in each case. As expected, ${ }^{[1,36]}$ one notices that the main characteristics of the forward and backward voltammetric peaks were not severely altered, especially for the slow charge transfer case, but that the most noticeable improvement of this down-scaling was to eliminate the trends to introduce partial steady-state contributions that produce larger currents compared to pure Cottrellian ones onto the two post-peak tails and restore correct Cottrellian behavior. ${ }^{[1,36]}$

\section{Cylindrical approximation of unit cells}

Correct prediction of the individual site/electrode contributions is extremely important when a material with non-ordered distribution of active sites needs to be analyzed on the basis of its electrochemical response (see e.g. ref.[32]). However, as can be testified by the present work and previous ones, a rigorous prediction of the electrochemical behavior of any irregular or random array requires heavy/expensive computational means and theoretical skills that altogether are often disproportionate with respect to the ultimate goal of the sought solution. In this respect, validating the use of much simpler approximation is still an important issue.

Among the simplest ways for treating theoretically such systems is that originally introduced by one of us $^{[1]}$ for regular hexagonal arrays of nanodisk electrodes embedded in an insulating substrate and which was later further implemented to treat random arrays. ${ }^{[12,28,29,31]}$ It consists in representing the elementary cells by cylindrical cells with symmetry axes perpendicular to the insulating material, and inside which the electroactive disc electrodes are located at the center of the circular bases of surface areas $A_{c y l}$. Provided that its validity is confirmed, which has never been done for voltammetry, this approach is indeed extremely effective since, thanks to the cylindrical symmetry, it allows converting 3D simulations into 2D ones. Moreover, it may allow to implement even faster 1D-semi-analytical predictions based on the analogy of the corresponding physico-mathematical equations governing the transport and boundary conditions with CEC mechanisms. ${ }^{[1,37]}$

For such cylindrical unit cells, $A_{\text {cell }}^{\text {Voronoi }}$ and $A_{\text {cross }}$ are identical by definition to $A_{\text {cyl }}$. However, one must decide which value should be given to $A_{c y l}$ in order to model the voltammetric behavior of one unit cell within a random array. One may impose that $A_{\text {cyl }}=A_{\text {cell }}^{\text {Voronoi }}$ and use a set of $A_{\text {cell }}^{\text {Voronoi values }}$ based on a statistical analysis of the array when possible as was proposed in previous studies. ${ }^{[28-32]} \mathrm{We}$

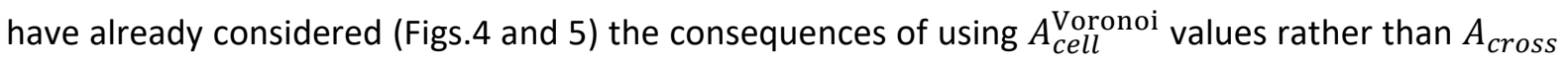
ones and shown that while it leads to almost correct predictions for the overall voltammetric currents, its results in terms of the individual ones for each unit cells are totally wrong. This could therefore introduce a serious problem including for the global current for electrochemical systems in which the amplitudes of the local concentration gradients can have an important mechanistic influence as occurs for example during electrodimerizations or even more complex mechanisms. Accordingly, we do not 
report here the corresponding investigations made using $A_{c y l}=A_{\text {cell }}^{\text {Voronoi }}$.

Conversely, we have established above that the use of $A_{\text {cross }}$ values is well suited to represent the voltammetric behaviors of the unit cells as well as that of the overall elementary cell. Therefore, using $A_{\text {cyl }}=A_{\text {cross }}$ seems more suitable than using $A_{\text {cyl }}=A_{\text {cell }}^{\text {Voronoi }}$. However, in an actual experimental situation, the $A_{\text {cross }}$ values cannot be known without recording the individual CVs for each electroactive disk, leading to a circular loop and total experimental nonsense. Nevertheless, we established above (see e.g., Table 1 ) that for a given array of disk electrodes, the $A_{\text {cross }}$ values tend to converge within ca. $10 \%$ towards a common mean value $A_{c y l}=A_{\text {array }} / 3$ where $A_{\text {array }}$ is the total surface area of the array of $N=3$ disk electrodes in Fig.1c and Table 1. Therefore, one may think of arbitrarily enforcing $A_{c y l}=A_{\text {array }} / N$ for each equivalent unit cylindrical cell irrespective of its exact Voronoi tessellate surface area $A_{\text {cell }}^{\text {Voronoi }}$. Note that, even if the purpose of assuming this was different as explained in the Introduction, this is exactly what was considered in ref.[1].
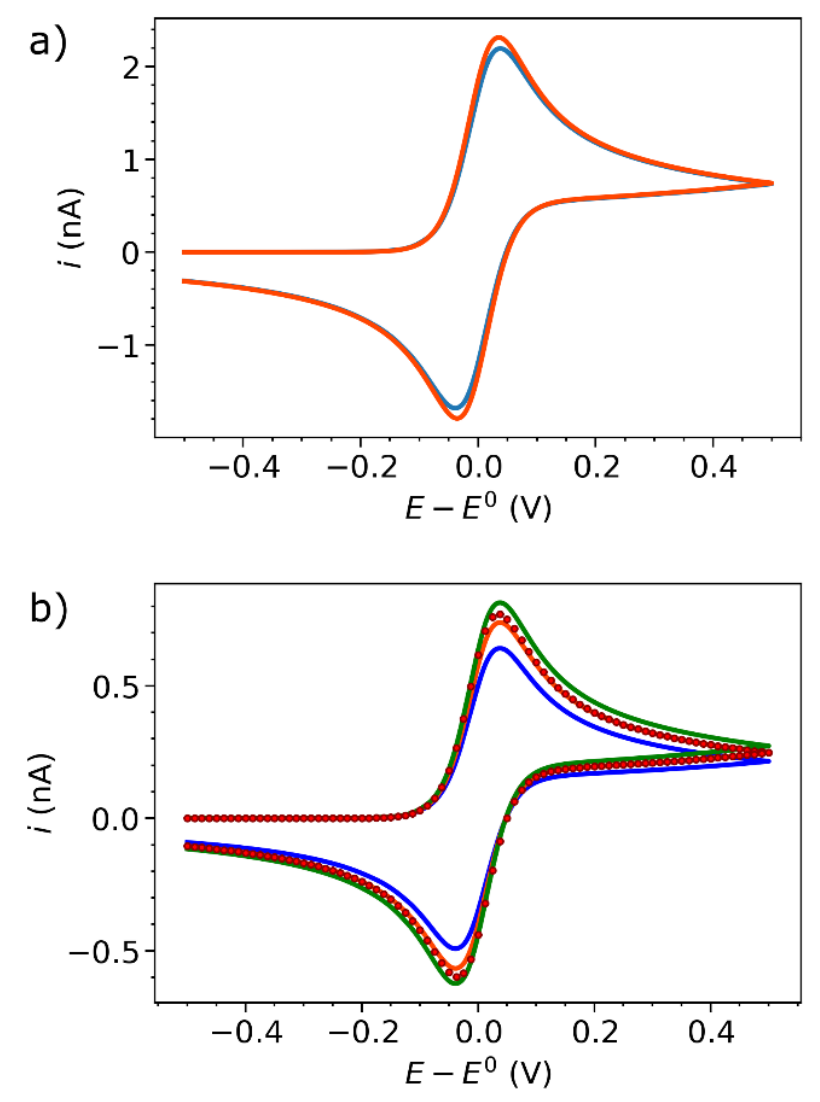

Figure 8. Comparison of the CVs for the array of disk electrodes in Fig.1c at $v=$ $10^{-3} \mathrm{~V} / \mathrm{s}$ for $k_{0}=10^{2} \mathrm{~cm} / \mathrm{s}$ predicted from simulations: a) total currents (blue whole elementary cell; orange - equivalent array of three cylindrical cells with $A_{c y l}=$ $A_{\text {array }} / 3$ ) or b) electrode-wise currents for whole elementary cell simulations (solid lines) or for the equivalent array of three cylindrical cells (symbols) with $A_{c y l}=$ $A_{\text {array }} / 3$ (see text). In b) blue, orange and green lines relate to the currents flowing through electrodes 1, 2 and 3 (Fig.1C,d), respectively. All other parameters including arrays geometrical characteristics were as summarized in the Computational details section. 

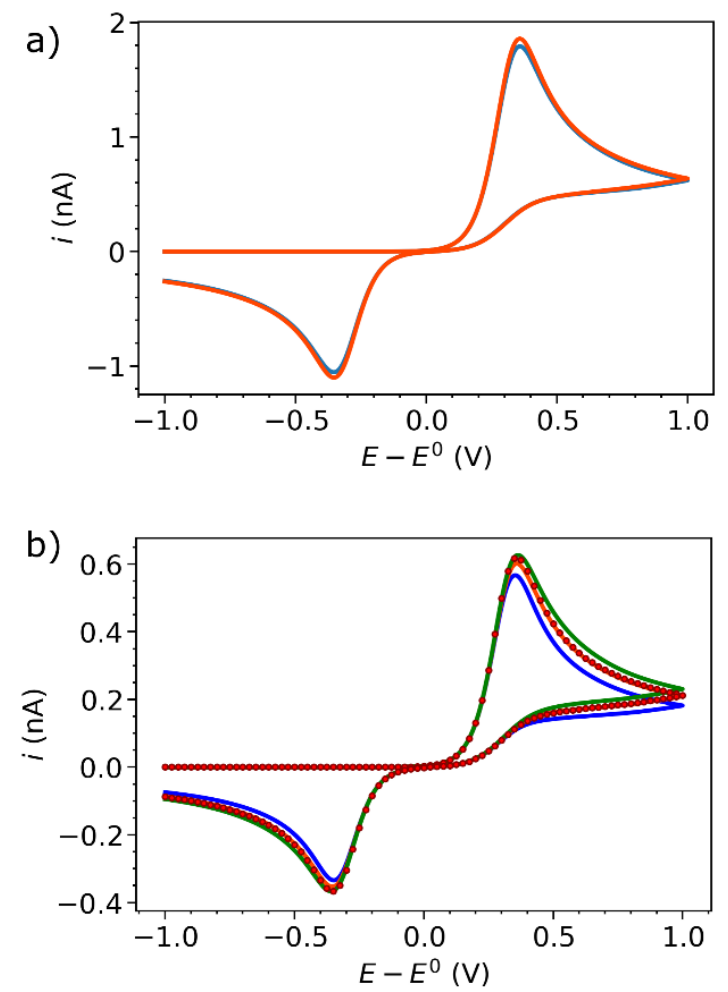

Figure 9. Comparison of the CVs for the array of disk electrodes in Fig.1c at $v=$ $10^{-3} \mathrm{~V} / \mathrm{s}$ for $k_{0}=10^{-5} \mathrm{~cm} / \mathrm{s}$ predicted from simulations a) total currents (blue whole elementary cell; orange - equivalent array of three cylindrical cells with $A_{c y l}=$ $A_{\text {array }} / 3$ ) or b) electrode-wise currents for whole elementary cell simulations (solid lines) or for the equivalent array of three cylindrical cells (symbols) with $A_{c y l}=$ $A_{\text {array }} / 3$ (see text). In b) blue, orange and green lines relate to the currents flowing through electrodes 1, 2 and 3 (Fig.1C,d), respectively. All other parameters including arrays geometrical characteristics were as summarized in the Computational details section.

Figures 8 and 9 test the validity of this systematic approximation for the two simple ET mechanisms with the rate constants considered in this study and for an elementary cell (Fig1c) having the same geometric dimensions as reported in the Computational Details section together with the values of the other parameters. It is observed that the replacement of each elementary cell 1-3 of Fig.1c by three identical cylindrical elementary cells whose base surfaces are $A_{c y l}=A_{\text {array }} / 3$ provides very good predictions (maximum deviation at peak currents ca. 5.5\% for fast kinetics and $3.7 \%$ for slow one, see Figs.8a and 9a) of the overall voltammetric behaviors of the array. Importantly, this result can be obtained based on the number of electrodes in the array without knowing their exact geometrical positions. The quality of these predictions needs to be compared to those $(2.6 \%$ and $4.8 \%$, respectively) based on a Voronoi approach but it must be noted that defining the corresponding tessellations on the array surface requires a priori an exact knowledge of each electrode position. At the level of the individual electrode responses one notices larger deviations for unit cells $1(-13.0 \%)$ and $3(+11.0 \%)$, which are coherent with the values of $A_{\text {cross }}^{(1)}=0.29 A_{\text {array }}$ and $A_{\text {cross }}^{(3)}=0.37 A_{\text {array }}$ (Table 1 , Figs.8b and $9 \mathrm{~b}$ ) relative to that of $A_{c y l}=A_{\text {array }} / 3$. Conversely, one notice a better agreement for the unit cell $2(+2.0 \%)$, in line with the fact that $A_{\text {cross }}$ value for this unit cell is $A_{\text {cross }}^{(2)}=$ 
$0.34 A_{\text {array }}$ (Table 1), is close to the common $A_{c y l}$ value. Importantly, for our purpose here, these deviations need to be compared to the much larger ones $(-43.0 \%,-7.0 \%$ and $+50.0 \%)$ obtained respectively for unit cells 1,2, and 3 based on Voronoi tessellation (Table 1, Figs.4b and 5b). Finally, it is worthwhile to underline that although the real unit cell 2 and its cylindrical or Voronoi equivalents have almost identical basal surfaces (Table 1), one could nevertheless have expected to observe more significant differences since in the equivalent cylindrical cell the electroactive disk is perfectly centered on the vertical axis of the cell while Figure $1 \mathrm{c}$ shows that this is not the case for the actual unit cell 2 . In fact, this result is perfectly consistent with those we have already established in a previous work focused on the chronoamperometric responses of random arrays to investigate the consequences related to these off-centering problems, ${ }^{[28]}$ and validates the high quality of the cylindrical approximations even when one knows exact positions of the active sites in an array as soon as the voltammetric wave resemble a classical one.

Finally, given the excellent predictive properties of the cylindrical cells analogy, it should be mentioned at this point that the voltammetric responses of the arrays whose dimensions were down-scaled by factors of 10 and 100 (Figures 6 and 7) took advantage of these equivalences. This made it possible to reduce the duration of the simulations which would have been necessary for complete simulations (16 $\mathrm{h}$ each!) of the corresponding systems.

\section{Conclusions}

Almost 40 years after the seminal work of Amatore and Savéant, ${ }^{[1,12]}$ we can finally conclude in complete theoretical safety that in the absence of any precise independent information on the exact geometry of a random array of electrodes of identical discs, an approach considering that it behaves like $N$ identical independent cylindrical unit cells of basal surface area $A_{c y l}=A_{\text {array }} / N$ would allow correct predictions or an adequate rationalization of the experimental CVs as soon as these CVs present characteristics close to those expected for a flat electrode with minimal involvement of current components visible in steady state or quasi-stable, that is, provided that the representative system is on the right side of the zone diagram shown in Figure 6. As carefully established this a priori counter-intuitive behavior stems from the competitive interaction between the diffusion layers that are generated by the activity of each individual electroactive site that leads to a near identity of the cross-sections of the bulk solution that "feed" each of these sites.

This conclusion explains a posteriori why the theory developed by Amatore and Savéant for regular arrays and the corresponding approximation assuming that its voltammetric behavior is to that afforded by identical independent cylindrical unit cells performing in parallel could be profitably used and applied to the treatment of arrays that were random by definition.

\section{Acknowledgements}

This work was supported in parts by CNRS, ENS - PSL University and Sorbonne University (UMR 8640 PASTEUR). GP acknowledges CNRS Institute of Chemistry (INC) for the "Emergence" post-doctoral support. AO thanks CNRS INC for the award of the "Emergence@INC2020" grant. CA acknowledges the Sino-French LIA CNRS NanoBioCatEchem for the support, as well as Xiamen University for his Distinguished Visiting Professor position. 


\section{References:}

[1] C. Amatore, J.-M. Savéant, D. Tessier, J. Electroanal. Chem. 1983, 147, 39-51.

[2] R. Landsberg, R. Thiele, Electrochim. Acta. 1966, 11, 1243-1259.

[3] F. Scheller, S. Muller, R. Landsberg, H.J. Spitzer, J. Electroanal. Chem. 1968, 19, 187-198.

[4] F. Scheller, R. Landsberg, S Muller, J. Electroanal. Chem. 1969, 20, 375-381.

[5] R. Guidelli, G. Piccardi, Anal. Chem. 1971, 43, 1639-1645.

[6] L. Nadjo, J.M. Savéant, J. Electroanal. Chem. Interfacial Electrochem. 1973, 48, 113-145.

[7] Basic Concepts. C. Amatore, in "Organic Electrochemistry, Fifth Edition: Revised and Expanded", (O. Hammerich, B. Speiser, Eds.). CRC Press, Taylor \& Francis Group, Boca Raton (FL, USA), 2016, Chap. 1. pp. 3-96.

[8] C. Amatore, J.-M. Savéant, D. Tessier, J. Electroanal. Chem. 1983, 146, 37-45.

[9] E. Sabatani, I. Rubinstein, R. Maoz, J. Sagiv, J. Electroanal. Chem. Interf. Electrochem. 1987, $219,365-371$.

[10] E. Sabatani, I. Rubinstein, J. Phys. Chem. 1987, 91, 6663-6669.

[11] R.M. Penner, C.R. Martin, Anal. Chem. 1987, 59, 2625-2630.

[12] C. Amatore, Electrochemistry at ultramicroelectrodes, in: I. Rubinstein (Ed.), Physical Electrochemistry: Principles, Methods and Applications, M. Dekker, New York, 1995, p. 131. Ch. 4.

[13] For a recent review, see, e.g.: M. Ongaro, P. Ugo, Anal. Bioanal. Chem. 2013, 405, 3715-3729.

[14] J. Kim, C. Renault, N. Nioradze, N. Arroyo-Curras, K.C. Leonard, A.J. Bard, J. Am. Chem. Soc. 2016, $138,8560-8568$.

[15] M. Nesselberger, M. Roefzaad, R.F. Hamou, P.U. Biedermann, F.F. Schweinberger, S. Kunz, K. Schloegl, G.K.H. Wiberg, S. Ashton, U. Heiz, K.J.J. Mayrhofer, M. Arenz, Nat. Mater. 2003, 12, 919.

[16] S.R. Belding, R.G. Compton, J. Phys. Chem. C. 2010, 114, 8309-8319.

[17] M.C. Henstridge, R.G. Compton, Chem Record. 2012, 12, 63-71.

[18] O. Sliusarenko, A. Oleinick, I. Svir, C. Amatore, Electroanalysis 2015, 27, 980-991.

[19] R.C. Engstrom, K.W. Johnson, S. DesJarlais, Anal. Chem. 1987, 59, 670-673.

[20] R.C. Engstrom, C.M. Pharr, M.D. Koppang, J. Electroanal. Chem. Interf. Electrochem. 1987, 221, 251-255.

[21] C.M. Pharr, R.C. Engstrom, R.A. Tople, T.K. Bee, P.L. Unzelman, J. Electroanal. Chem. Interf. Electrochem. 1990, 278, 119-128.

[22] R.C. Engstrom, S. Ghaffari, H. Qu, Anal. Chem. 1992, 64, 2525-2529.

[23] S. Szunerits, J.M. Tam, L. Thouin, C. Amatore, D.R. Walt, Anal. Chem. 2003, 75, 4382-4388.

[24] C. Amatore, C. Pebay, L. Servant, N. Sojic, S. Szunerits, L. Thouin, ChemPhysChem 2006, 7, 1322-1327. 
[25] C. Amatore, A. Oleinick, O.V. Klymenko, L. Thouin, L. Servant, I. Svir, ChemPhysChem 2007, 8, 1664-1676.

[26] C. Pebay, C. Sella, L. Thouin, C. Amatore, Anal. Chem. 2013, 85, 12062-12069.

[27] J.-F. Lemineur, J.-M. Noël, C. Combellas, F. Kanoufi, J. Electroanal. Chem. 2020, 872, 114043.

[28] O. Sliusarenko, A. Oleinick, I. Svir, C. Amatore, ChemElectroChem 2015, 2, 1279-1291.

[29] T.J. Davies, R.G. Compton, J. Electroanal. Chem. 2005, 585, 63-82.

[30] S.R. Belding, E.J.F. Dickinson, R.G. Compton, J. Phys. Chem. C 2009, 113, 11149-11156.

[31] A. Oleinick, O. Sliusarenko, I. Svir, C. Amatore, J. Electrochem. 2017, 23, 141-158.

[32] A. Oleinick, O. Sliusarenko, I. Svir, C. Amatore, J. Electrochem. Soc. 2020, 167, 013530.

[33] M.E. Hyde, R.G. Compton, J. Electroanal. Chem. 2003, 549, 1-12.

[34] G. Pireddu, I. Svir, C. Amatore, A. Oleinick, Electrochim. Acta. 2021, 365, 137338.

[35] E. Laviron, J. Electroanal. Chem. 1979, 101, 19-28.

[36] A.C. Michael, R.M. Wightman, C. Amatore, J. Electroanal. Chem. 1989, 267, 33-45.

[37] See,e.g.,: Y.R. Leroux, P. Hapiot, Chem. Mater. 2013, 25, 489-495. 